\title{
Performance of novel tissue harmonic echo imaging using endoscopic ultrasound for pancreatic diseases
}

Authors

Institutions
Kazuyuki Matsumoto ${ }^{1, *}$, Akio Katanuma ${ }^{1, *}$, Hiroyuki Maguchi ${ }^{1}, K^{\prime}$ uniyuki Takahashi', Manabu Osanai ${ }^{1}$, Kei Yane ${ }^{1}$, Toshifumi Kin', Ryo Takaki', Tomoaki Matsumori', Katsushige Gon ${ }^{1}$, Akiko Tomonari', Masanori Nojima ${ }^{2}$

${ }^{1}$ Center for Gastroenterology, Teine-Keijinkai Hospital, Hokkaido, Japan

${ }^{2}$ Center for Translational Research, The Institute of Medical Science, The University of Tokyo, Tokyo, Japan

\section{Bibliography}

DOI http://dx.doi.org/

10.1055/s-0034-1393367

Published online: 19.11.2015

Endoscopy International Open

2016; 04: E42-E50

(c) Georg Thieme Verlag KG

Stuttgart · New York

E-ISSN 2196-9736

\section{Corresponding author}

Kazuyuki Matsumoto, MD

Center for Gastroenterology

Teine-Keijinkai Hospital

1-jo 12-chome, Maeda,

Teine-ku

Sapporo 006-8555

Japan

Phone: +81-11-681-8111

Fax: +81-11-685-2967

matsumotokazuyuki0227@

yahoo.co.jp
Background and study aims: Recently, tissue harmonic echo (THE) imaging has advanced with the development of a new endoscopic ultrasound (EUS) monitor/processing unit. With this new technology, penetration (THE-P) and resolution (THE-R) images can be obtained. The aim of this study was to investigate the performance of this novel THE imaging using a new processing unit for pancreatic diseases.

Patients and methods: Fifty patients with pancreatic lesions (38 cystic, 12 solid) were retrospectively analyzed. At each examination, 3 EUS images of the same pancreatic lesion were obtained using B-mode, THE-P mode, and THE-R mode imaging. Each set of EUS images was randomly arranged and evaluated independently by 4 physicians blinded to the imaging technique. Images were compared using a Likert scale 5-point grading system for each parameter.

\section{Introduction}

$\nabla$

Tissue harmonic echo (THE) imaging is a sonographic technique that can potentially provide higher-quality images than conventional B-mode imaging. On THE imaging, higher harmonic frequencies generated by ultrasound beam propagation through tissue are used to produce the sonogram. Its potential advantages include enhanced resolution, improved signal-to-noise ratio, and reduced artifacts [ $1-4]$.

THE imaging has been used clinically during transabdominal ultrasonography (US), and its usefulness for diagnosing several organ diseases has been reported $[5,6]$. However, the diagnostic utility of transabdominal US for pancreatic lesions is limited by the anatomic location of the pancreas. To address this, endoscopic ultrasound (EUS) is used for closer examination of pancreatic

\section{License terms}

\section{()(1) $\Theta \circledast$}

\footnotetext{
* These authors contributed equally.
}

Results: For cystic lesions, THE-P mode images were significantly superior to conventional Bmode images for visualizing the boundary, septum, nodules, and total image quality $(P<0.05)$. THE-R mode images were significantly superior to conventional B-mode images for visualizing the boundary, septum, and total image quality $(P$ $<0.05$ ). However, for solid lesions, there was no significant difference in all the evaluation points between THE-P and conventional B-mode images. THE-R mode images were inferior to conventional B-mode images for visualizing the boundary, internal structure, and total image quality $(P<0.05)$. Conclusions: For pancreatic cystic lesions, THE mode images provided better lesion characterization than conventional B-mode images. Further research is required to determine if this improvement will result in improved EUS diagnostics.

lesions. EUS is thus indispensable for the investigation and management of pancreatic diseases [7-9].

Recently, THE imaging has advanced with the development of a new EUS monitor/processing unit $(\bullet$ Fig. 1). With this new technology, penetration (THE-P) and resolution (THE-R) images can be obtained. THE-P mode imaging may be suitable for middle-range observation because it receives a harmonic signal with a frequency of $7.5 \mathrm{MHz}$. THE-R mode imaging may be suitable for closedistance observation because it receives a harmonic signal with a frequency ranging from 10 to $12 \mathrm{MHz}$. We previously reported the usefulness of novel THE imaging techniques for intraductal papillary mucinous neoplasm (IPMN) with mural nodules [10].

To date, only a few studies have evaluated pancreatic diseases by THE imaging using EUS $[4,11]$. We evaluated the performance of the novel THE imaging for pancreatic diseases. 


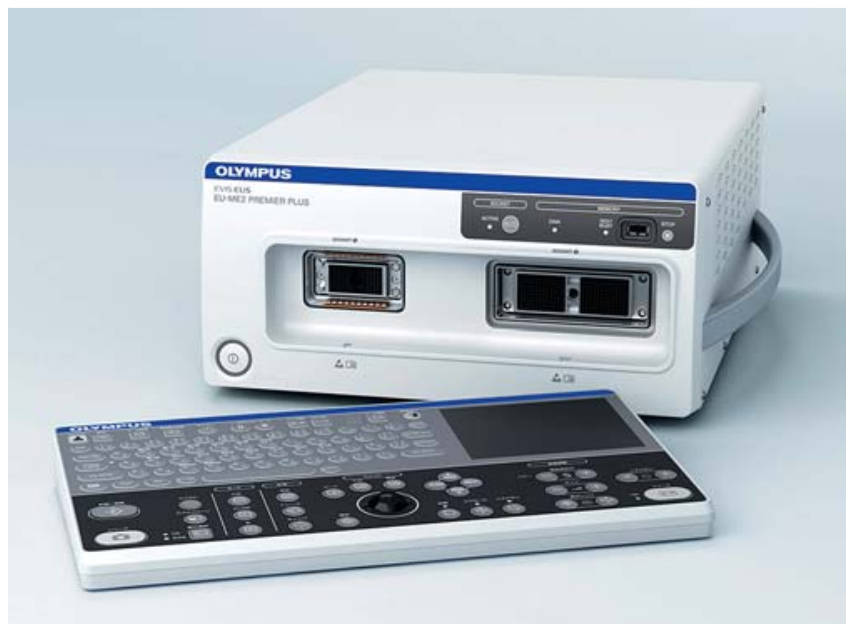

Fig. 1 The EU-ME2 is a high-quality compact ultrasound processor for use with endoscopic ultrasound equipment that has been designed for integration with conventional endoscopy on a single workstation. It is possible to use new functions such as Tissue Harmonic Echo (THE), Contrast Harmonic EUS (CH-EUS) and Elastography.

\section{Patients and methods}

\section{$\nabla$}

Between September 1, 2013 and March 1, 2014, 50 patients with pancreatic solid or cystic lesions who underwent EUS with a newly developed monitor/processing unit at our hospital were retrospectively analyzed. Of these patients, 38 had cystic lesions and 12 had solid lesions.

Patients with cystic lesions were followed up by computed tomography, magnetic resonance imaging, or EUS for at least 6 months. The clinical course and radiologic findings were compatible for each disease. Patients with solid lesions were confirmed by surgical resection or endoscopic ultrasound-guided fine needle aspiration (EUS-FNA). This study was approved by the Institutional Review Board of our hospital.

\section{Image acquisition}

EUS was performed using 2 types of endoscope, radial scanning (EU-260; Olympus, Tokyo, Japan) or curved linear array scanning (UCT-260; Olympus), and a newly developed monitor/processing unit (EU-ME2 PREMIER PLUS; Olympus) equipped with THE technology.

With this imaging system, it is possible to change between Bmode and THE mode in real time. In each examination, 3 EUS images of the same pancreatic lesion were obtained using Bmode, THE-P mode, and THE-R mode imaging. The imaging parameters were identical for each patient, except image gain which was optimized for each image. EUS examinations were performed by well-experienced endoscopists and images were stored digitally.

\section{Image analysis}

EUS images acquired by THE-P mode, THE-R mode, and B-mode imaging were evaluated independently by 4 physicians with extensive EUS experience (reviewer 1: 4000 procedures; 2: 3000 procedures; 3: 1500 procedures; 4: 1200 procedures) and who had not performed the actual EUS examinations. Each set of EUS images obtained using 3 sonographic techniques was randomly arranged with no indication of which technique was used.
The reviewers viewed the images on a computer monitor screen and evaluated their ability to visualize features in each set as follows: in patients with cystic lesions, boundary $(n=38)$, septum $(n=32)$, nodules $(n=8)$, and total image quality $(n=38)$; in patients with solid lesions, boundary $(n=12)$, internal structures $(n=12)$, and total image quality $(n=12)$. For the boundary, septum, and internal structures, the reviewers evaluated their ability to visualize the proximal and distal sides of each lesion. The proximal side image was defined as the area from the EUS probe to the lesion center. The distal side image indicated the area from the lesion center to the far side. The average distances from the probe to the lesion center were $14.1 \pm 6.1 \mathrm{~mm}$ (cystic lesion) and $15.5 \pm 7.6 \mathrm{~mm}$ (solid lesion).

Finally, we compared the total image quality of each mode in lesions whose distance from the EUS probe to the lesion center was within $1 \mathrm{~cm}$, less than $2 \mathrm{~cm}$, more than $1 \mathrm{~cm}$, and more than $2 \mathrm{~cm}$ to evaluate the characteristics of each mode. In lesions within $1-\mathrm{cm}$ distance, the average distance was $7.6 \pm 1.6 \mathrm{~mm}$ in the cystic lesion and $8.0 \pm 2.1 \mathrm{~mm}$ in the solid lesion. In lesions less than $2 \mathrm{~cm}$ but more than $1-\mathrm{cm}$ distance, the average distance was $13.4 \pm 2.6 \mathrm{~mm}$ in the cystic lesion and $12.4 \pm 1.8 \mathrm{~mm}$ in the solid lesion. In lesions with more than 2 -cm distance, the average distance was $24.0 \pm 5.7 \mathrm{~mm}$ in the cystic lesion and $24.5 \pm 6.3 \mathrm{~mm}$ in the solid lesion. We also analyzed the correlation between each mode grade for total image quality and distance from the EUS probe to the lesion center. THE-P mode, THE-R mode, and B-mode images were compared using a Likert scale 5-point grading system as follows: grade 1 , "very poor image"; grade 2 , "poor image"; grade 3, "moderate image”; grade 4, "good image”; grade 5, "excellent image” for each parameter.

\section{Statistical analysis}

SPSS 11.0.1 J (SPSS, Chicago, IL) was used for the statistical analysis. The Wilcoxon signed rank test was used to evaluate the scores for the evaluation criteria. $P$-values $<0.05$ were considered to indicate a significant difference. Bonferroni's correction was used to adjust for multiple testing within each evaluation.

The inter-rater reliability between the 4 reviewers for grading each parameter was assessed using the coefficient of variation (CV). The CV was calculated from the average value of each parameter obtained by each reviewer. Percent CV values were considered to represent a close agreement defined as less than 20.

\section{Results \\ $\nabla$}

\section{Patient characteristics}

The clinical characteristics of the 50 patients with pancreatic solid or cystic lesions who underwent EUS are shown in $\bullet$ Table 1. Of these patients, 38 had cystic lesions and 12 had solid lesions. Their median age was 68 years (range, 40 - 86), and there were 32 female patients. As for lesion location, 23 lesions were located in the pancreatic head, 22 in the body, and 5 in the tail. The median size of the pancreatic lesions was $27 \mathrm{~mm}$ (range, 10-60).

The patients with cystic lesions comprised 34 cases of intraductal papillary mucinous neoplasm (IPMN), 3 cases of serous cystic neoplasm, and 1 case of lymphoepithelial cyst. The median follow-up period of the patients with the cystic lesions was 28 months (range, 6-108). 


\begin{tabular}{|llll|}
\hline Outcome measures & Overall & Cystic lesion & Solid lesion \\
\hline No. of patients & 50 & 38 & 12 \\
\hline Age, median (range), y & $68(40-86)$ & $68(40-86)$ & $71(50-83)$ \\
\hline Sex, female (\%) & $32(64)$ & $23(60.5)$ & $9(75)$ \\
\hline Location, no (\%) & & & $6(50)$ \\
\hline Head & 23 & $17(44.7)$ & $4(33.3)$ \\
\hline Body & 22 & $18(47.4)$ & $2(16.7)$ \\
\hline Tail & 5 & $3(7.9)$ & $25(12-60)$ \\
\hline Size, median (range), mm & $27(10-60)$ & $29(10-60)$ & - \\
\hline Etiology, no (\%) & & $34(89.5)$ & - \\
\hline IPMN & & 7 & - \\
\hline \multicolumn{1}{c}{ IPMN with nodule } & & $3(7.9)$ & $11(91.7)$ \\
\hline SCN & & $1(2.6)$ & $1(8.3)$ \\
\hline LEC & - & - & \\
\hline Pancreatic cancer & & & \\
\hline Metastatic cancer (RCC) & &
\end{tabular}

Table 1 Clinical characteristics of the patients with pancreatic cystic or solid lesions

IPMN, intraductal papillary mucinous neoplasm; SCN, serous cystic neoplasm; LEC, lymphoepithelial cyst; RCC, renal cell carcinoma

\begin{tabular}{|c|c|c|c|}
\hline \multirow[t]{2}{*}{ Outcome measures } & \multicolumn{3}{|c|}{ Sonographic mode } \\
\hline & B-mode & THE-P mode & THE-R mode \\
\hline \multicolumn{4}{|l|}{ Boundary (38 patients, 152 assessments) } \\
\hline Whole image & $2.97 \pm 1.06$ & $4.05 \pm 0.71$ & $3.61 \pm 0.97$ \\
\hline Proximal & $2.95 \pm 0.94$ & $4.04 \pm 0.75$ & $3.74 \pm 0.94$ \\
\hline Distal & $3.02 \pm 1.04$ & $3.99 \pm 0.75$ & $3.35 \pm 1.01$ \\
\hline \multicolumn{4}{|l|}{ Septum (32 patients, 128 assessments) } \\
\hline Whole image & $2.82 \pm 0.97$ & $3.96 \pm 0.77$ & $3.18 \pm 0.98$ \\
\hline Proximal & $2.88 \pm 0.98$ & $3.95 \pm 0.75$ & $3.25 \pm 1.03$ \\
\hline Distal & $2.89 \pm 1.02$ & $3.74 \pm 0.80$ & $3.04 \pm 1.06$ \\
\hline Nodule ( 7 patients, 28 assessments) & $2.89 \pm 0.98$ & $4.32 \pm 0.66$ & $3.46 \pm 0.94$ \\
\hline Total image quality (38 patients, 152 assessments) & $2.97 \pm 0.99$ & $4.17 \pm 0.71$ & $3.45 \pm 0.93$ \\
\hline
\end{tabular}

Table 2 Average grading by 4 reviewers for boundary, septum, nodule, and total image quality of pancreatic cystic lesions

\begin{tabular}{|l|l|}
\hline Outcome measures & Better sonographic technique \\
\hline $\begin{array}{l}\text { Boundary (38 patients, } 152 \text { assessments) } \\
\text { Whole image }\end{array}$ & THE-P mode $>$ THE-R mode $>$ B-mode \\
\hline Proximal & THE-P mode $>$ THE-R mode $>$ B-mode \\
\hline Distal & THE-P mode $>$ THE-R mode $>$ B-mode \\
\hline Septum (32 patients, 128 assessments) & THE-P mode $>$ THE-R mode $>$ B-mode \\
\hline Whole image & THE-P mode $>$ THE-R mode $>$ B-mode \\
\hline Proximal & THE-P mode $>$ THE-R mode $=$ B-mode \\
\hline Distal & THE-P mode $>$ THE-R mode $=$ B-mode \\
\hline Nodule (7 patients, 28 assessments) & THE-P mode $>$ THE-R mode $>$ B-mode \\
\hline Total image quality ( 38 patients, 152 assessments) & \\
\hline
\end{tabular}

Table 3 Comparative statistical analysis of images obtained using 3 techniques in pancreatic cystic lesions

Data were analyzed using the Wilcoxon signed rank test with Bonferroni's correction.

$\mathrm{A}>\mathrm{B}$ : $\mathrm{A}$ is better than $\mathrm{B}$ with statistical significance $(P<0.05)$

$A=B$ : No statistically significant difference between $A$ and $B$

$A=B$ : No statistically significant difference between $A$ and $B$

The patients with solid lesions comprised 11 cases of pancreatic cancer and 1 metastatic mass from renal cell carcinoma (RCC). Of these 11 cases, 2 were confirmed by surgical resection and the remaining 9 by EUS-FNA. The metastatic mass from RCC was confirmed by EUS-FNA.

\section{Evaluation for pancreatic cystic lesions \\ Boundary}

Thirty-eight cystic lesions comprising 152 assessments were evaluated. In all the evaluation points of boundary, both THE-P mode and THE-R mode images were significantly superior to Bmode images $(P<0.05)(\otimes$ Table $2, \bullet$ Table 3$)$.

\section{Septum}

Thirty-two lesions comprising 128 assessments were evaluated. On whole and proximal images, both THE-P and THE-R modes were significantly superior to $\mathrm{B}$-mode $(P<0.05)$. On distal images, THE-P mode was significantly superior to B-mode $(P<0.05)$; the difference between THE-R mode and B-mode imaging was not significant.

Nodules

Seven lesions comprising 28 assessments were evaluated. On nodule images, THE-P mode was significantly superior to B-mode $(P<0.05)$; the difference between THE-R mode and B-mode imaging was not significant. 

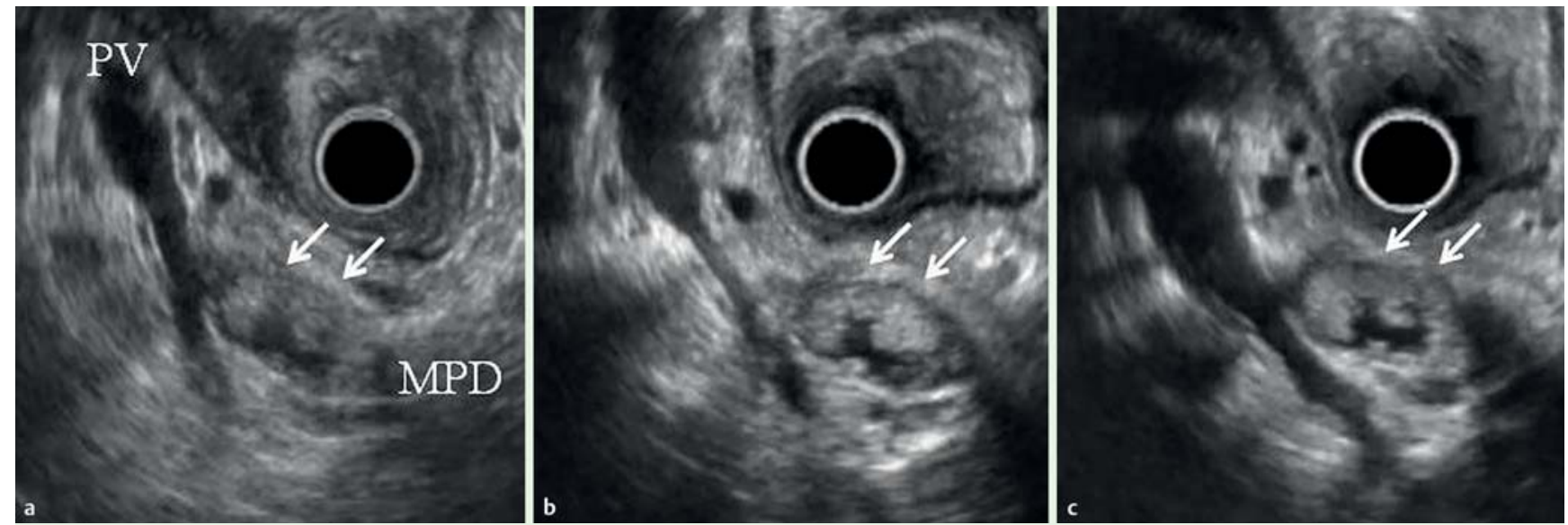

Fig.2 Main duct intraductal papillary mucinous neoplasm of the pancreas in a 67-year-old woman. a Conventional B-mode imaging showed mural nodules (arrows) in the dilated main pancreatic duct (MPD) of the pancreatic body (arrows). b THE-P mode imaging showed mural nodules (arrows) in the dilated MPD more clearly than B-mode. c THE-R mode imaging clearly showed mural nodules (arrows) in the dilated MPD, similarly to the THE-P mode imaging.
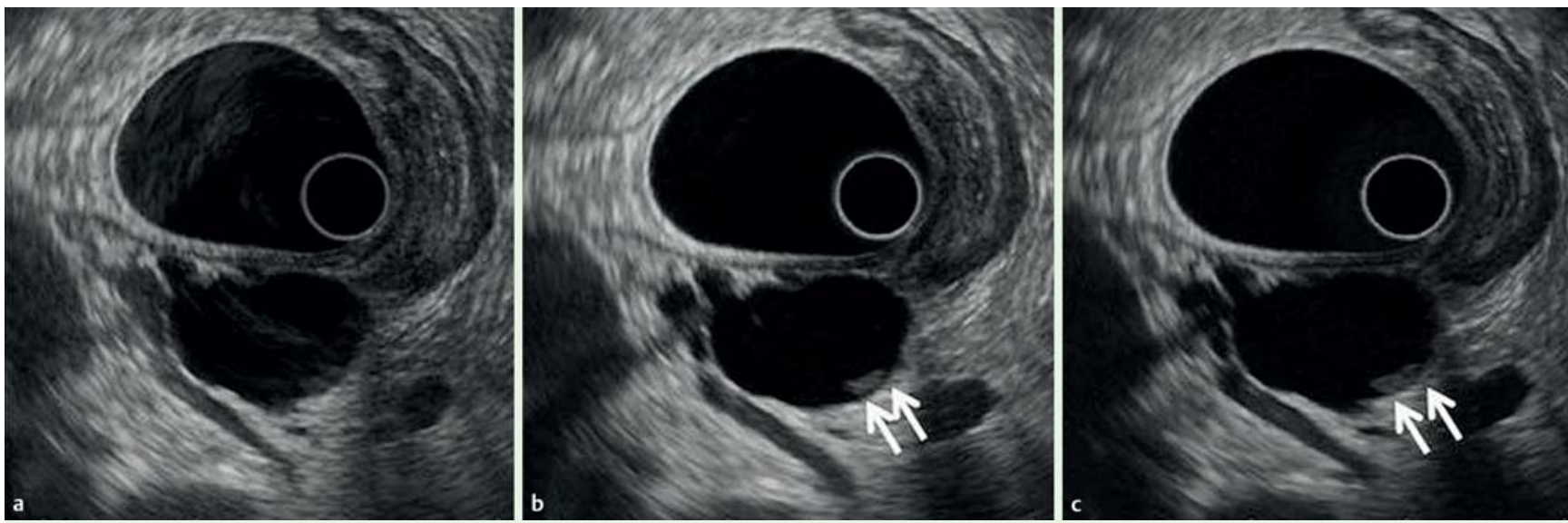

Fig. 3 Intraductal papillary mucinous neoplasm of the pancreatic body in a 78-year-old woman. a Conventional B-mode imaging could not clearly detect the mural nodule in the cyst. b THE-P mode imaging revealed a mural nodule (arrows) in the cyst and more clearly visualized the boundary and total image. c THE-R mode imaging demonstrated a mural nodule (arrows), similarly to THE-P mode imaging.

Total image quality

Thirty-eight lesions comprising 152 assessments were evaluated. In total image quality, both THE-P and THE-R modes were significantly superior to B-mode $(P<0.05)$. Still and video images show a cystic lesion evaluated using the 3 techniques $(\bullet$ Fig. 2, $\odot$ Fig. 3, - Fig.4, $\odot$ Video 1 ).

\section{Evaluation for pancreatic solid lesions \\ Boundary}

Twelve lesions comprising 48 assessments were evaluated $\bullet$ Table 4, $\odot$ Table 5. On whole and distal images, B-mode imaging was significantly superior to THE-R mode imaging $(P<0.05)$; the difference between B-mode and THE-P mode imaging was not significant. On proximal images, B-mode was significantly superior to both THE-P and THE-R modes $(P<0.05)$.

\section{Internal structure}

Twelve lesions comprising 48 assessments were evaluated. In all the evaluation points of internal structure, B-mode images were significantly superior to THE-R mode images $(P<0.05)$. The differences between $\mathrm{B}$-mode and THE-P mode images were not significant.
Total image quality

Twelve lesions comprising 48 assessments were evaluated. For total image quality, B-mode was significantly superior to THE-R mode $(P<0.05)$. The difference between B-mode and THE-P mode imaging was not significant ( $\bullet$ Fig. 5 ).

Evaluation for total image quality of each distance from EUS probe

Pancreatic cystic lesions

Lesions within $1-\mathrm{cm}$ distance Of the 38 cystic lesions, 8 had a distance of less than $1 \mathrm{~cm}$ from the EUS probe to the lesion center. The order of the average grade for total image quality from highest to lowest was THE-P mode $(4.13 \pm 0.70)$, THE-R mode ( $3.56 \pm$ $0.80)$, and B-mode $(2.84 \pm 0.88)$. Both THE-P and THE-R modes were significantly superior to $B$-mode $(p<0.05)$.

Lesions with less than $2-\mathrm{cm}$ but more than $1-\mathrm{cm}$ distance Of the 38 cystic lesions, 23 had a distance of less than $2 \mathrm{~cm}$ but more than $1 \mathrm{~cm}$ from the EUS probe to the lesion center. The order of the average grade for total image quality from highest to lowest was THE-P mode (4.16 \pm 0.73$)$, THE-R mode ( $3.60 \pm 0.95)$, and Bmode $(2.92 \pm 0.99)$. Both THE-P and THE-R modes were significantly superior to B-mode $(P<0.05)$. 

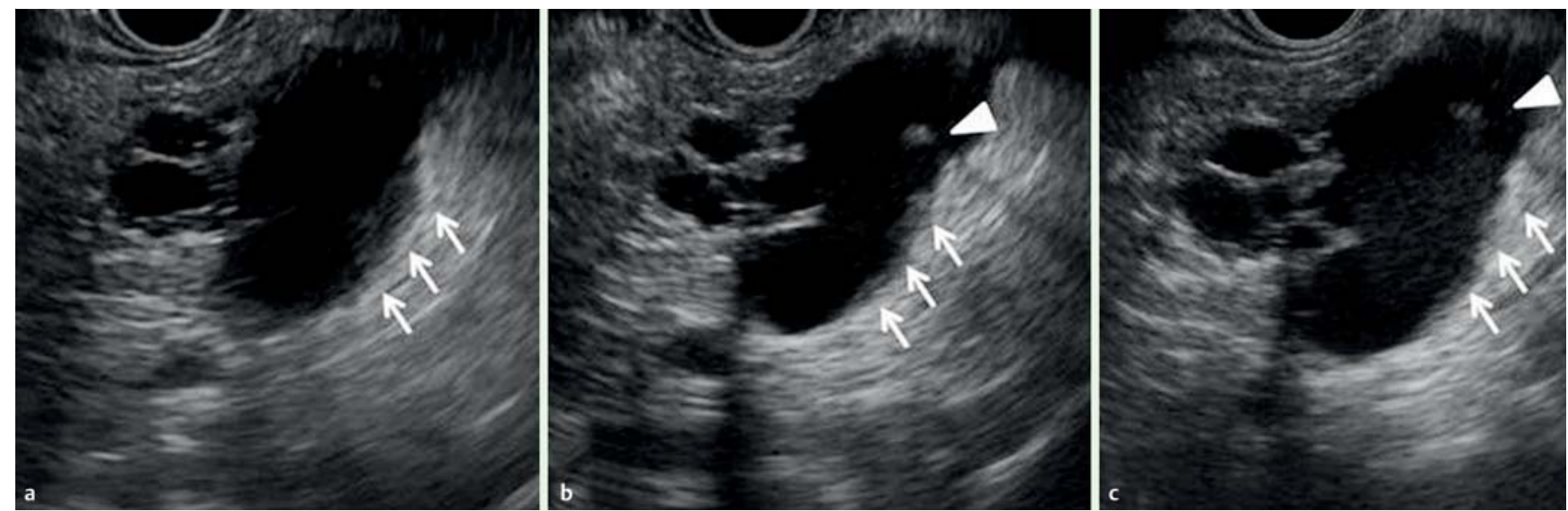

Fig.4 Intraductal papillary mucinous neoplasm of the pancreatic body in a 51-year-old man. a Conventional B-mode imaging could not clearly visualize the thickening of the septum (arrows) and nodule in the cyst. b THE-P mode imaging more clearly visualized the thickening of the septum (arrows) and nodule (arrowhead) in the cyst. c THE-R mode imaging was better than conventional B-mode imaging; however, it was slightly less clear than THE-P mode imaging.

\section{Video 1}

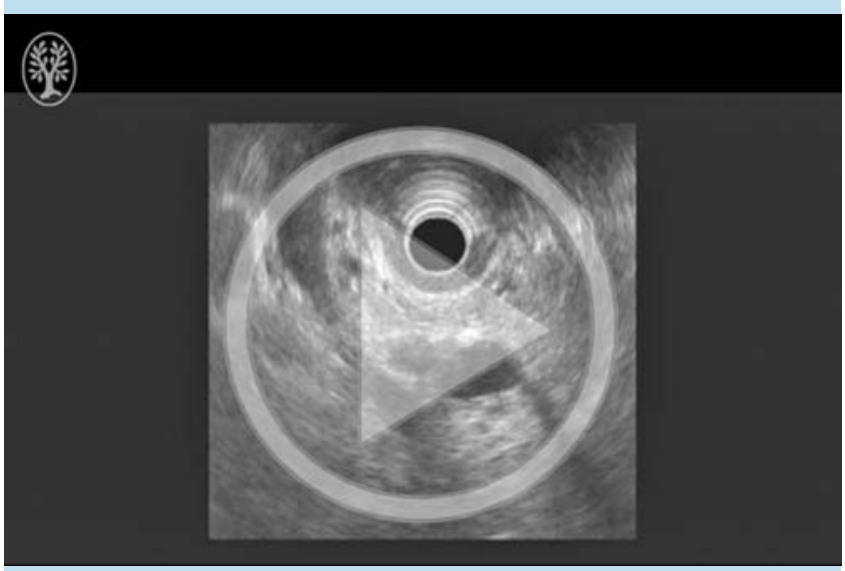

Main duct intraductal papillary mucinous neoplasm of the pancreas in a 67-year-old woman. These images were observed from around the antrum of the stomach using a radial type of EUS. Conventional B-mode imaging showed mural nodules in the dilated MPD of the pancreatic body. THE-P mode imaging visualized mural nodules in the dilated MPD more clearly than B-mode imaging. THE-R mode imaging clearly visualized mural nodules in the dilated MPD similarly to the THE-P mode imaging. Online content including video sequences viewable at: http://dx.doi.org/10.1055/s0034-1393367

Lesions with more than 2-cm distance of the 38 cystic lesions, 7 had a distance of more than $2 \mathrm{~cm}$ from the EUS probe to the lesion center. The order of the average grade for total image quality from highest to lowest was THE-P mode (4.25 \pm 0.65$)$, B-mode $(3.25 \pm 1.11)$, and THE-R mode $(2.86 \pm 0.80)$. THE-P mode was significantly superior to B-mode $(P<0.05)$; there was no significant difference between THE-R mode and B-mode imaging.

Correlation between mode grade and distance ( $\bullet$ Fig. 6 ) The grade for total image quality of B-mode was not significantly correlated with the distance from the EUS probe to the lesion center $(\mathrm{r}=0.011, P=0.17)$. The grade of THE-P mode was also not significantly correlated with the distance from the EUS probe to the lesion center $(r=0.062, P=0.45)$. The grade of THE- $R$ mode was in- versely and weakly correlated with the distance from the EUS probe to the lesion center $(r=-0.22, P=0.0058)$.

\section{Pancreatic solid lesions}

Lesions within 1-cm distance of the 12 lesions, 3 had a distance of less than $1 \mathrm{~cm}$ from the EUS probe to the lesion center. The order of the average grade for total image quality from highest to lowest was B-mode ( $3.67 \pm 0.89)$, THE-P mode ( $3.41 \pm 0.90)$, and THE$\mathrm{R}$ mode ( $3.33 \pm 0.89)$. There was no significant difference between B-mode and THE modes.

Lesions with less than $2-\mathrm{cm}$ but more than $1-\mathrm{cm}$ distance of the 12 lesions, 5 had a distance of less than $2 \mathrm{~cm}$ but more than $1 \mathrm{~cm}$ from the EUS probe to the lesion center. The order of the average grade for total image quality from highest to lowest was THE-P mode (3.80 \pm 0.89$)$, B-mode ( $3.75 \pm 0.85)$, and THE-R mode (2.80 $\pm 0.89)$. B-mode was significantly superior to THE-R mode $(P<$ $0.05)$. The difference between THE-P mode and B-mode was not significant.

Lesions with more than 2-cm distance of the 12 lesions, 4 had a distance of more than $2 \mathrm{~cm}$ from the EUS probe to the lesion center. The order of the average grade for total image quality from highest to lowest was B-mode ( $3.63 \pm 0.96)$, THE-P mode ( $3.50 \pm$ $1.03)$, and THE-R mode $(2.56 \pm 0.73)$. B-mode was significantly superior to THE-R mode $(P<0.05)$. The difference between THEP mode and B-mode was not statistically significant ( $\bullet$ Fig. 7). Correlation between mode grade and distance ( $\bullet$ Fig.8) The grade of total image quality of B-mode was not significantly correlated with the distance from the EUS probe to the lesion center $(\mathrm{r}=-0.047, P=0.76)$. The grade of THE-P mode was also not significantly correlated with the distance from the EUS probe to the lesion center $(r=0.027, P=0.86)$. The grade of THE-R mode was inversely and weakly correlated with the distance from the EUS probe to the lesion center $(r=-0.37, P=0.0092)$.

\section{Inter-rater reliability}

The inter-rater reliability between the 4 reviewers for grading each parameter is summarized in Table6. For cystic lesions, the $\% \mathrm{CV}$ values were $5.1 \%-7.7 \%$. For solid lesions, the $\% \mathrm{CV}$ values were $13.0 \%-17.3 \%$. For solid lesions, the $\%$ CV values were slightly higher; however, the total average \% CV was $10.4 \%$, which was considered to represent a close agreement. 


\begin{tabular}{|c|c|c|c|}
\hline \multirow[t]{2}{*}{ Outcome measures } & \multicolumn{3}{|c|}{ Sonographic mode } \\
\hline & B-mode & THE-P mode & THE-R mode \\
\hline \multicolumn{4}{|l|}{ Boundary ( 12 patients, 48 assessments) } \\
\hline Whole image & $3.77 \pm 0.80$ & $3.58 \pm 0.98$ & $2.96 \pm 0.87$ \\
\hline Proximal & $3.79 \pm 0.79$ & $3.33 \pm 0.96$ & $3.38 \pm 0.95$ \\
\hline Distal & $3.58 \pm 0.86$ & $3.63 \pm 0.95$ & $2.54 \pm 0.96$ \\
\hline \multicolumn{4}{|l|}{ Internal structure (12 patients, 48 assessments) } \\
\hline Whole image & $3.56 \pm 0.89$ & $3.83 \pm 0.94$ & $2.67 \pm 0.75$ \\
\hline Proximal & $3.58 \pm 0.89$ & $3.69 \pm 0.92$ & $2.90 \pm 0.74$ \\
\hline Distal & $3.38 \pm 1.01$ & $3.65 \pm 0.97$ & $2.48 \pm 0.89$ \\
\hline Total image quality ( 12 patients, 48 assessments) & $3.69 \pm 0.87$ & $3.60 \pm 0.93$ & $2.85 \pm 0.87$ \\
\hline
\end{tabular}

Table 4 Average grading by 4 reviewers for boundary, internal structure, and total image quality of pancreatic solid lesions

\begin{tabular}{|l|l|}
$\begin{array}{l}\text { Outcome measures } \\
\text { Boundary (12 patients, } 48 \text { assessments) }\end{array}$ & Better sonographic technique \\
\hline $\begin{array}{l}\text { Whole image } \\
\text { Proximal }\end{array}$ & B-mode $=$ THE-P mode $>$ THE-R mode \\
\hline Distal & B-mode $>$ THE-R mode $=$ THE-P mode \\
\hline Internal structure (12 patients, 48 assessments) & THE-P mode $=$ B-mode $>$ THE-R mode \\
\hline Whole image & THE-P mode $=$ B-mode $>$ THE-R mode \\
\hline Proximal & THE-P mode $=$ B-mode $>$ THE-R mode \\
\hline Distal & THE-P mode $=$ B-mode $>$ THE-R mode \\
\hline Total image quality (12 patients, 48 assessments) & B-mode $=$ THE-P mode $>$ THE-R mode \\
\hline
\end{tabular}

Table 5 Comparative statistical analysis of images obtained using 3 techniques in pancreatic solid lesions

Data were analyzed using the Wilcoxon signed rank test with Bonferroni's correction.

$A>B$ : A is better than $B$ with statistical significance $(P<0.05)$

$A=B$ : No statistically significant difference between $A$ and $B$

$A=B$ : No statistically significant difference between $A$ and $B$
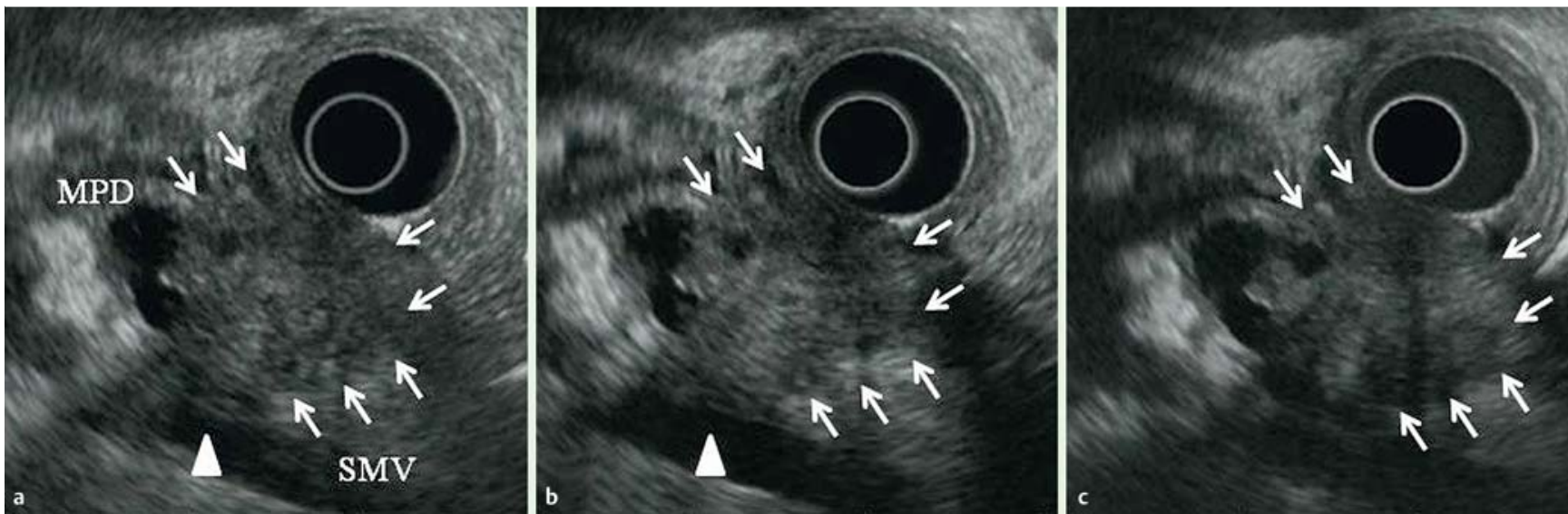

Fig. 5 An 83-year-old woman with cancer of the pancreatic head. a Conventional B-mode imaging clearly revealed the boundary of the solid lesion (arrows). The pancreatic cancer caused an obstruction in the main pancreatic duct (MPD) and invaded the superior mesenteric vein (SMV) (arrowhead). b THE-P mode imaging clearly demonstrated the boundary and internal structure, similarly to B-mode imaging. C THE-R mode imaging was not superior to the other imaging techniques, particularly because the distal image produced was poor.
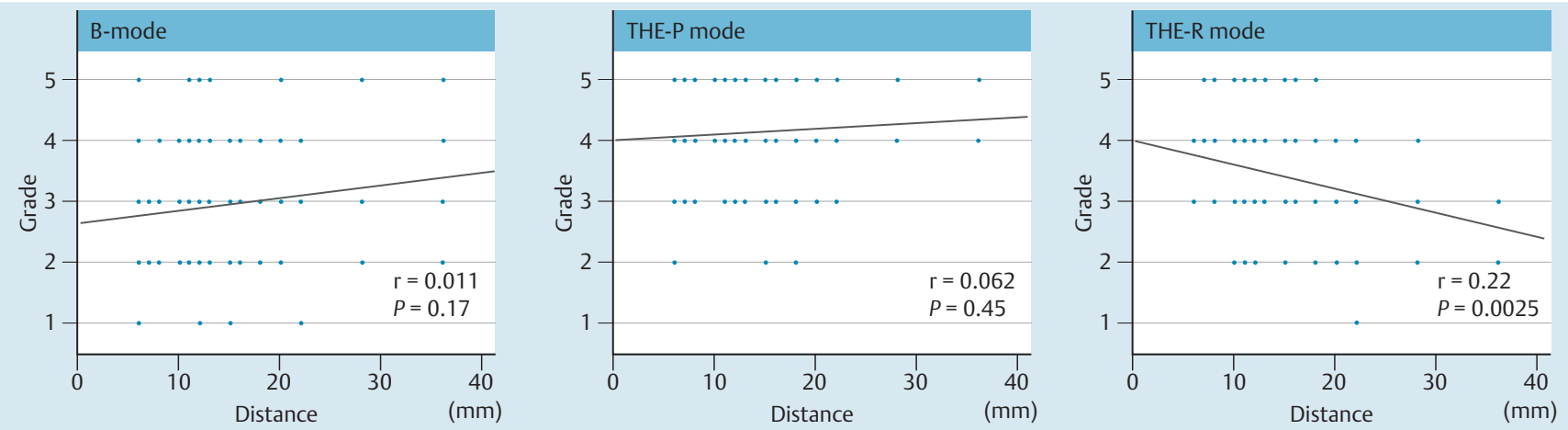

Fig. 6 Correlation between mode grade and distance in pancreatic cystic lesions The grade for the total image quality of B-mode was not significantly correlated with the distance from the EUS probe to the center of the lesion $(r=0.011, P=0.17)$. The grade of THE-P mode was also not significantly correlated with the distance from the EUS probe to the center of the lesion $(r=0.062, P=0.45)$. The grade of THE-R mode was inversely and weakly correlated with the distance from the EUS probe to the center of the lesion $(r=-0.22, P=0.0058)$. 

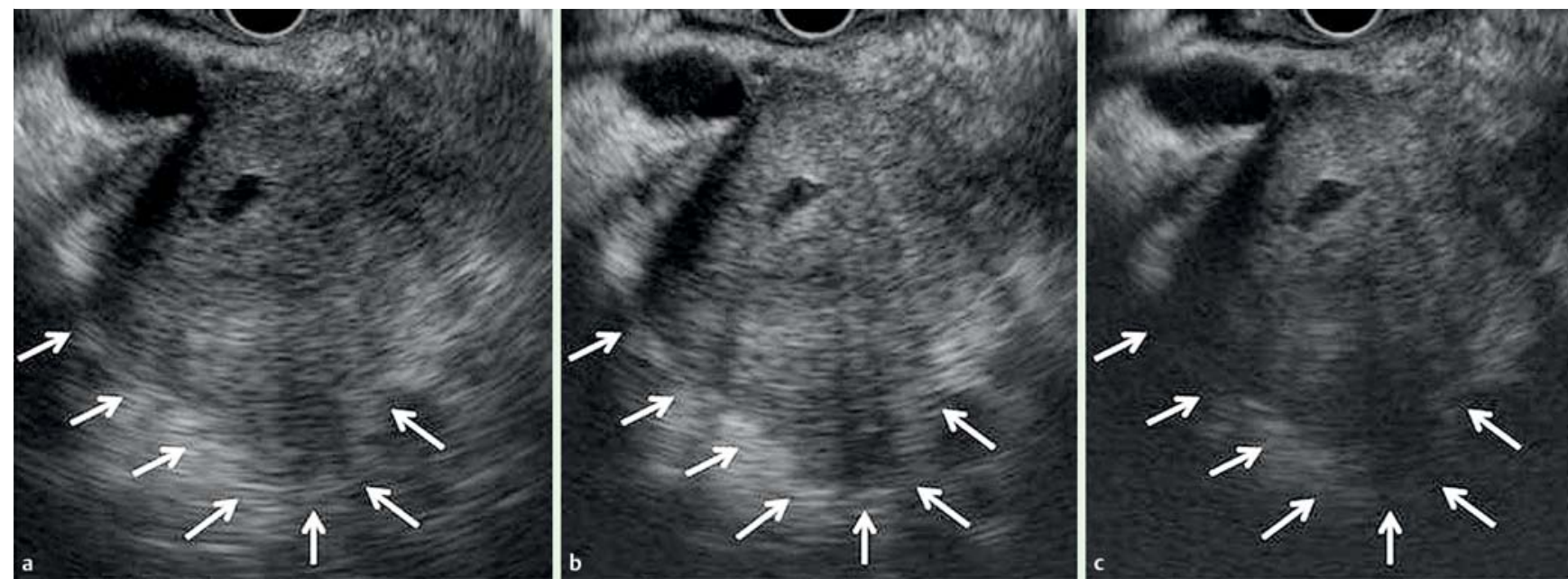

Fig.7 A 72-year-old man with cancer of the pancreatic head. The distance from the EUS probe to the lesion center was $3.5 \mathrm{~cm}$. a Conventional B-mode imaging could visualize the distal side of the boundary of the lesion (arrows). b THE-P mode imaging could visualize the distal side of the boundary of the lesion, similar to B-mode imaging (arrows). c THE-R mode imaging could not clearly visualize the distal side of the boundary of the lesion (arrows).
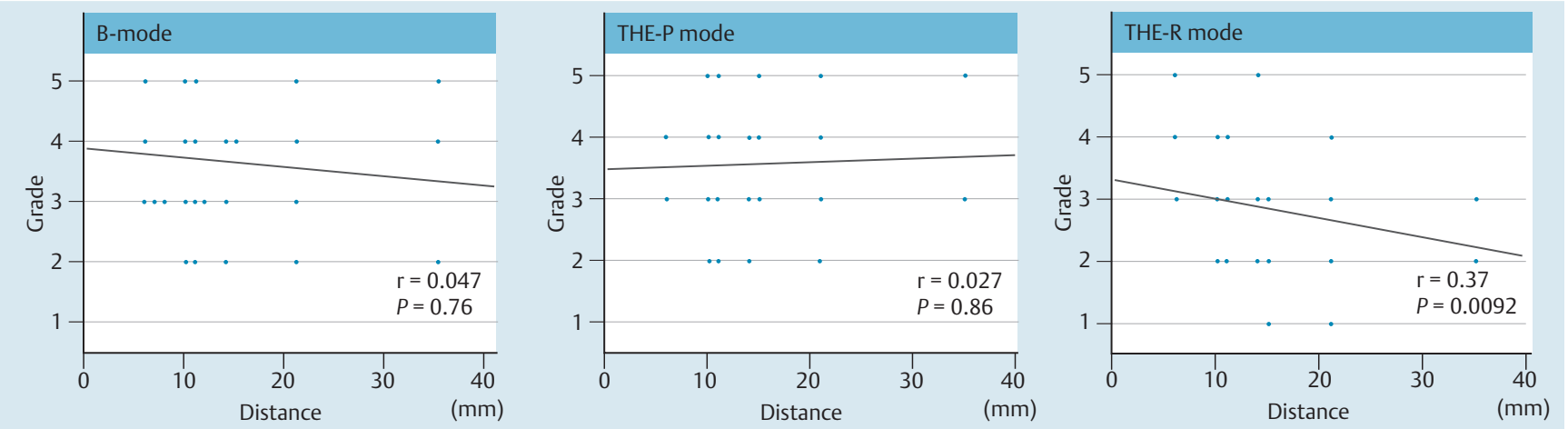

Fig. 8 Correlation between mode grade and distance in pancreatic solid lesions

The grade of the total image quality of B-mode was not significantly correlated with the distance from the EUS probe to the center of the lesion ( $r=-0.047$, $P=0.76)$. The grade of THE-P mode was also not significantly correlated with the distance from the EUS probe to the center of the lesion $(r=0.027, P=0.86)$. On the other hand, the grade of THE-R mode was inversely and weakly correlated with the distance from the EUS probe to the center of the lesion ( $r=-0.37$, $P=0.0092)$.

\section{Discussion}

$\nabla$

Since its introduction, EUS has had a significant impact on the diagnosis of pancreatic diseases. High-resolution imaging of the pancreas is achievable owing to the close proximity between the luminal structures and the pancreas. Moreover, EUS findings are not affected by the presence of gas in the bowel loops. Thus, EUS is essential for diagnosing pancreatic lesions and is an accurate method for diagnosing pancreatic diseases [7-9].

THE imaging is a means of visualizing the harmonic components in the form of an image. In the harmonic components, a large amount of the main beam is generated. The harmonic components make it possible to view the beam advancing in the forward direction, thereby minimizing artifacts [ $1-4]$. These THE imaging characteristics significantly improve image quality. The current results showed that in pancreatic cystic lesions, THE mode imaging improved the visualization of the boundary, septum, nodule, and total image quality compared with conventional B-mode imaging. THE mode imaging has been reported to be more advantageous for visualizing cystic lesions [4] as the anechoic area is clearly recognized. In solid lesions, THE mode imaging showed no improvement in the evaluated parameters compared with conventional B-mode imaging. The effectiveness of THE mode imaging for visualization of the boundary and internal structure has also been reported for pancreatic solid lesions [4]. Our study included a rather small number of pancreatic solid lesions, and most cases were pancreatic cancer. Notably, the difference between THE mode and B-mode imaging was not significant.

THE-R mode is suitable for observation at a close distance from the EUS probe because it receives harmonic signals with frequencies of 10 to $12 \mathrm{MHz}$. THE-P mode is suitable for observation at a middle-range distance from the EUS probe because it receives harmonic signals with frequencies of approximately $7.5 \mathrm{MHz}$. In our evaluation of the distal images of the cystic lesion septum, THE-P mode was significantly superior to B-mode, but the difference between THE-R mode and B-mode was not significant. The evaluation of the total image quality of THE-R mode showed an inverse correlation between the distance from the EUS probe and the grade. However, the grades of both B-mode and THE-P mode images were not correlated with the distance. In cystic lesions whose centers were more than $2 \mathrm{~cm}$ from the EUS probe, the average grade of THE-R mode was decreased from 3.45 (overall) to 2.86. The average grade of B-mode was increased from 2.97 (overall) to 3.25. Although THE-R mode was significantly superior to B-mode in the evaluation of overall lesions, there was no significant difference between THE-R mode and B-mode imaging 
Table 6 Average grading by 4 reviewers for each outcome measure and \% CV between 4 reviewers

\begin{tabular}{|c|c|c|c|c|c|}
\hline \multirow[t]{2}{*}{ Outcome measures } & \multicolumn{4}{|l|}{ Reviewer } & \multirow[t]{2}{*}{$\% \mathrm{CV}$} \\
\hline & 1 & 2 & 3 & 4 & \\
\hline \multicolumn{6}{|l|}{ Cystic lesion } \\
\hline \multicolumn{6}{|l|}{ Boundary (38 patients, 114 assessments) } \\
\hline Whole image & $3.77 \pm 0.98$ & $3.68 \pm 1.00$ & $3.27 \pm 0.93$ & $3.45 \pm 1.23$ & 6.4 \\
\hline Proximal & $3.80 \pm 0.97$ & $3.75 \pm 0.97$ & $3.37 \pm 0.90$ & $3.39 \pm 1.06$ & 6.4 \\
\hline Distal & $3.59 \pm 1.07$ & $3.67 \pm 1.01$ & $3.20 \pm 0.83$ & $3.36 \pm 1.09$ & 6.2 \\
\hline \multicolumn{6}{|l|}{ Septum (32 patients, 96 assessments) } \\
\hline Whole image & $3.50 \pm 1.20$ & $3.44 \pm 1.15$ & $3.21 \pm 0.87$ & $3.15 \pm 1.01$ & 5.1 \\
\hline Proximal & $3.55 \pm 1.07$ & $3.46 \pm 1.14$ & $3.28 \pm 0.91$ & $3.14 \pm 0.93$ & 5.5 \\
\hline Distal & $3.31 \pm 1.05$ & $3.39 \pm 1.13$ & $3.16 \pm 0.93$ & $3.04 \pm 0.99$ & 4.8 \\
\hline Nodule ( 7 patients, 21 assessments) & $3.90 \pm 0.97$ & $3.48 \pm 1.10$ & $3.24 \pm 1.11$ & $3.62 \pm 0.90$ & 7.7 \\
\hline \multirow[t]{2}{*}{ Total image quality (38 patients, 114 assessments) } & $3.83 \pm 0.88$ & $3.68 \pm 0.95$ & $3.25 \pm 1.00$ & $3.36 \pm 1.10$ & 7.7 \\
\hline & \multicolumn{5}{|c|}{ Total score of each reviewer for cystic lesion ${ }^{1}: 1=2>3=4$} \\
\hline \multicolumn{6}{|l|}{ Solid lesion } \\
\hline \multicolumn{6}{|l|}{ Boundary ( 12 patients, 36 assessments) } \\
\hline Whole image & $3.81 \pm 0.94$ & $3.69 \pm 0.99$ & $3.50 \pm 0.65$ & $2.75 \pm 0.79$ & 13.8 \\
\hline Proximal & $3.83 \pm 0.90$ & $3.72 \pm 0.99$ & $3.61 \pm 0.76$ & $2.83 \pm 0.68$ & 13.0 \\
\hline Distal & $3.58 \pm 1.11$ & $3.69 \pm 1.02$ & $3.08 \pm 0.76$ & $2.64 \pm 0.92$ & 14.9 \\
\hline \multicolumn{6}{|l|}{ Internal structure (12 patients, 36 assessments) } \\
\hline Whole image & $3.86 \pm 0.95$ & $3.78 \pm 0.92$ & $3.14 \pm 0.85$ & $2.63 \pm 0.71$ & 17.3 \\
\hline Proximal & $3.81 \pm 0.94$ & $3.75 \pm 0.95$ & $3.31 \pm 0.62$ & $2.69 \pm 0.61$ & 15.3 \\
\hline Distal & $3.64 \pm 1.11$ & $3.56 \pm 1.07$ & $2.97 \pm 0.90$ & $2.50 \pm 0.80$ & 16.9 \\
\hline Total image quality (12 patients, 36 assessments) & $3.78 \pm 0.97$ & $3.81 \pm 0.88$ & $3.17 \pm 0.80$ & $2.78 \pm 0.79$ & 14.8 \\
\hline
\end{tabular}

2>3: 2 is better than 3 with statistical significance $(P<0.05)$

${ }^{1}$ Data were analyzed using the Wilcoxon signed rank test with Bonferroni's correction.

in the evaluation of lesions with more than $2 \mathrm{~cm}$ distance. In solid lesions with less than $1 \mathrm{~cm}$ distance from the EUS probe to the lesion center, the average grade of THE-R mode was increased from 2.85 (overall) to 3.33. Although B-mode was significantly superior to THE-R mode in the evaluation of the overall lesions, there was no significant difference between THE-R mode and B-mode imaging in the evaluation of lesions with a distance within $1 \mathrm{~cm}$. Therefore, THE-R mode is suitable for lesions located close to the EUS probe, and that THE-P mode is effective for both close and more distant lesions. In this study, however, the image quality of THE-R mode was not superior to that of THE-P mode in all the evaluation parameters. Thus, THE-R mode may be indicated for the observation of closer lesions such as mucosal lesions of the gastrointestinal tract or other pancreatobiliary lesions.

In pancreatic solid lesions, EUS can usually guide EUS-FNA for obtaining cytologic samples of pancreatic lesions, enabling pathologic diagnosis. For the diagnosis of pancreatic and bile duct disorders, the usefulness of THE, particularly THE-P mode, has been indicated [12]. When we performed EUS-FNA for pancreatic solid tumors with cystic changes, we could recognize the solid part in the lesion using THE mode. As a result, we could move the needle to reach the target site correctly. On the other hand, the differential diagnosis of pancreatic cystic lesions remains challenging. Recently, the efficacies of differential diagnosis and tumor grading of cystic lesions by cytologic and chemical analyses of cyst fluid using EUS-FNA have been reported [13]. However, care should be taken in sampling, particularly for malignant cystic lesions $[14,15]$. In this study, the new THE mode imaging improved visualization compared with the conventional B-mode imaging in pancreatic cystic lesions. Thus, THE mode imaging may be useful for obtaining a definitive EUS diagnosis.

This study had some limitations. First, this study was analyzed retrospectively and there was a bias of disease and a small num- ber of pancreatic solid lesions. Among the patients with cystic lesions, 34 (89.3\%) had IPMN. Among those with solid lesions, 11 (91.7\%) had pancreatic cancer. Further prospective studies including more pancreatic lesions are needed. Second, only the quality of EUS images was evaluated but not the diagnosis of pancreatic cystic or solid lesions. Additional studies including the diagnostic accuracy of pancreatic diseases are needed. Third, this study was performed at a single center with a small number of observers, and only highly experienced endosonographers were enlisted. The evaluation of EUS still imaging which was performed using a Likert scale 5-point grading system generated highly subjective data, thus the inter-rater reliability was not particularly high. Therefore, we defined \% CV values less than 20 to represent a close agreement. The total average \% CV was $10.4 \%$, which was considered to represent a close agreement. The \% CV values of cystic lesions were better than those of solid lesions. Thus, THE mode images may be suitable for cystic lesion evaluation. We also evaluated the inter-reviewer differences among four reviewers. In both cystic and solid lesions, the total scores of reviewer 1 and 2 were higher than those for reviewers 3 and 4. Reviewers 1 and 2 had more experience than did reviewers 3 and 4 . As for possible reasons, there may be a difference in experience. Multicenter prospective studies should be performed using a more objective evaluation method with good inter-rater reliability.

In conclusion, this study showed that EUS images obtained using the newly developed monitor/processing unit for THE imaging can provide better lesion characterization than conventional Bmode images for pancreatic cystic lesions. Further research is required to determine whether this improvement will result in improved EUS diagnostics.

\section{Competing interests: None}




\section{Acknowledgements}

The author are indebted to Dr. Edward Barroga, Associate Professor and Senior Medical Editor of the Department of International Medical Communications of Tokyo Medical University for reviewing and editing the English manuscript.

\section{References}

1 Tranquart F, Grenier N, Eder $V$ et al. Clinical use of ultrasound tissue harmonic imaging. Ultrasound Med Biol 1999; 256: 889-894

2 Ward B, Baker AC, Humphrey VF. Nonlinear propagation applied to the improvement of resolution in diagnostic medical ultrasound. J Acoust Soc Am 1997; 101: 143 - 154

3 Shapiro RS, Wagreich J, Rarsons RB et al. Tissue harmonic imaging sonography: evaluation of image quality compared with conventional sonography. Am J Roentgenol 1998; 171: $1203-1206$

4 Ishikawa $\mathrm{H}$, Hirooka $\mathrm{Y}$, Itoh $\mathrm{A}$ et al. A comparison of image quality between tissue harmonic imaging and fundamental imaging with an electronic radial scanning echoendoscope in the diagnosis of pancreatic diseases. Gastrointest Endosc 2003; 57: 931 -936

5 Kim SH, Lee JM, Kim KG et al. Comparison of fundamental sonography, tissue-harmonic sonography, fundamental compound sonography, and tissue-harmonic compound sonography for focal hepatic lesions. Eur Radiol 2006; 16: 2444-2453
6 Blaivas M, DeBehnke D, Sierzenski PR et al. Tissue harmonic imaging improves organ visualization in trauma ultrasound when compared with standard ultrasound mode. Acad Emerg Med 2002; 9: 48-53

7 Byrne MF, Jowell PS. Gastrointestinal imaging: Endoscopic ultrasound. Gastroenterology 2002; 122: 1631-48

8 Kahl S, Glasbrenner B, Zimmermann S et al. Endoscopic ultrasound in pancreatic disease. Dig Dis 2002; 20: 120 - 126

9 Meritz HR, Sechopoulos P, Delbeke D et al. EUS, PET, and CT scanning for evaluation of pancreatic adenocarcinoma. Gastrointest Endosc 2000; 52: $367-371$

10 Matsumoto K, Katanuma A. Novel tissue harmonic imaging clearly visualizes a case of intraductal papillary mucinous neoplasm with mural nodules. JOP 2014; 15: 03259-260

11 Bang JY, Hawes RH, Varadarajule S. Objective evaluation of a new endoscopic ultrasound processor. Dig Endosc 2013; 25: 05554-555

12 Katanuma A, Isayama $H$, Bapaye A. Endoscopic ultrasonography using new functions for pancreatobiliary disease: Current status and future perspectives. Dig Endosc 2015; 27: 47-54

13 Thosani N, Thosani S, Qiao W et al. Role of EUS-FNA-based cytology in the diagnosis of mucinous pancreatic cystic lesions: a systematic review and meta-analysis. Dig Dis Sci 2010; 55: 102756-2766

14 Yamao K, Mizuno N, Takagi T et al. How I do it and when I use (and do not use) EUS-FNA. Gastrointest Endosc 2009; 69: 134-137

15 Hirooka $Y$, Goto $H$, Itoh $A$ et al. Case of intraductal papillary mucinous tumor in which endosonography-guided fine-needle aspiration biopsy caused dissemination. J. Gastroenterol Hepatol 2003; 18: 13231324 